\title{
Triagem Auditiva Neonatal em crianças portadoras de sífilis congênita em Hospital de Aracaju em 2019
}

A audição é o principal sentido responsável pela aquisição da fala e linguagem da criança. A infecção por sífilis é responsável por uma alta taxa de morbimortalidade no período pré-natal e neonatal, podendo levar à hipoacusia neurossensorial precoce ou tardia. Neste estudo, objetiva-se analisar o perfil epidemiológico e fatores de riscos associados aos pacientes portadores de sífilis congênita que realizaram a Triagem Auditiva Neonatal (TAN) em um hospital de ensino na cidade de Aracaju no ano de 2019. Trata-se de um estudo transversal, analítico e retrospectivo com nascidos vivos portadores de sífilis congênita. 0 estudo avaliou 117 pacientes que foram acompanhados no ambulatório de sífilis congênita nesse hospital. Em 31 prontuários não havia informação sobre a realização da TAN e 26 recémnascidos (RN) não realizaram a triagem. Dos que fizeram o teste $69,8 \%$ ( $n=60$ ) cerca de $63,3 \%$ tiveram o resultado normal e $36,7 \%$ tiveram alterações auditivas. Entre os que apresentaram alteração auditiva, 54,5\% aguardavam a realização do reteste, $36,4 \%$ tinham reteste alterado e aguardavam uma nova avaliação e apenas $9,1 \%$ apresentou reteste normal. Ademais, $26,5 \%$ das mães afirmaram não terem feito tratamento para sífilis congênita durante a gestação, evidenciando um pré-natal não adequado. Por fim, a maioria dos recém-nascidos com sífilis congênita passou na TAN.

\section{Neonatal Hearing Screening in children with congenital syphilis in Aracaju Hospital in 2019}

\begin{abstract}
Hearing is the main sense responsible for the child's speech and language acquisition. Syphilis infection is responsible for a high rate of morbidity and mortality in the prenatal and neonatal period, which can lead to early or late sensorineural hearing loss. This study aims to analyze the epidemiological profile and risk factors associated with patients with congenital syphilis who underwent Neonatal Hearing Screening (NHS) at a teaching hospital in the city of Aracaju in 2019. This is a study cross-sectional, analytical and retrospective study with live births with congenital syphilis. The study evaluated 117 patients who were followed up at the congenital syphilis clinic at that hospital. In 31 medical records, there was no information about the NHS and 26 newborns (NB) did not perform the screening. Of those who took the test $69.8 \%(n=60)$ about $63.3 \%$ had a normal result and $36.7 \%$ had hearing disorders. Among those who had hearing impairment, $54.5 \%$ were waiting for the retest, $36.4 \%$ had an altered retest and were awaiting a new assessment and only $9.1 \%$ had a normal retest. In addition, $26.5 \%$ of mothers stated that they had not undergone treatment for congenital syphilis during pregnancy, showing an inadequate prenatal care. Finally, the majority of newborns with congenital syphilis passed NHS
\end{abstract}

Keywords: Hearing; Congenital Syphilis; Neonatal Screening

Topic: Pediatria e Saúde da Criança e do Adolescente

Reviewed anonymously in the process of blind peer.
Received: $19 / 10 / 2020$

Approved: 20/01/2021
Ana Maria dos Santos Gonçalves (iD

Universidade Tiradentes, Brasil

http://lattes.cnpq.br/6869356928151742

http://orcid.org/0000-0002-4322-2717

anamariasgoncalves@hotmail.com

Jordan de Oliveira Sousa Guimarães (iD)

Universidade Tiradentes, Brasil

http://lattes.cnpq.br/0532098780239721

http://orcid.org/0000-0002-6547-2856

jordan.de.o.s.guimaraes@gmail.com

Izailza Matos Dantas Lopes (iD

Universidade Tiradentes, Brasil

http://lattes.cnpq.br/5911269328493167

http://orcid.org/0000-0001-9752-5628

izailzamatos@gmail.com
Referencing this:

GONÇALVES, A. M. S.; GUIMARÂES, J. O. S.; LOPES, I. M. D.. Triagem Auditiva Neonatal em crianças portadoras de sífilis congênita em Hospital de Aracaju em 2019. Scire Salutis, v.11, n.1, p.69-75, 2021. DOI: http://doi.org/10.6008/CBPC2236-9600.2021.001.0008 


\section{INTRODUÇÃO}

Na primeira infância a criança depende essencialmente dos seus sentidos para comunicar-se com o mundo exterior e obter experiências que serão decisivas para as relações psicossociais. A perda auditiva tem implicações diretas no desenvolvimento, na escolaridade, no relacionamento social e no status emocional (WEBER et al., 2001). No que diz respeito à audição é fundamental que o sistema auditivo esteja íntegro anátomo e fisiologicamente a fim do desenvolvimento normal da linguagem oral (PÁDUA et al., 2005).

A deficiência auditiva é uma das alterações mais frequentes em recém-nascidos, ocorrendo em cerca de um a três neonatos para cada mil nascidos vivos. Quando se trata de neonatos que permaneceram na UTI, está prevalência é estimada entre um a quatro para cada cem nascidos vivos (BRASIL, 2012). Além disso, entre as doenças passíveis de serem identificadas ao nascimento, a deficiência auditiva apresenta elevada prevalência (30:10.000) quando comparada com a Fenilcetonúria (1:10.000), Hipotireoidismo (2,5:10.000) e Anemia Falciforme (2:10.000) (BRASIL, 2012).

As perdas auditivas no Brasil são diagnosticadas em média por volta dos dois a três anos de idade (RUGGIERI et al., 2002). Durante esse período, a criança perde informações auditivas importantes, o que interfere no desenvolvimento da sua comunicação.

As infecções congênitas (rubéola, sífilis, citomegalovírus, herpes, toxoplasmose e AIDS), mesmo quando assintomáticas, podem causar deficiência auditiva no neonato e podem estar associadas ao aparecimento tardio da perda auditiva e/ou à progressão da perda auditiva já existente ao nascimento (DAHLE et al., 2000; SANTOS et al., 2000; VIEIRA et al., 2005).

Analisar resultados da Triagem Auditiva Neonatal em maternidades constitui-se como fator importante para conhecer a prevalência de problemas auditivos e os seus fatores de risco (AZEVEDO, 2004). Diante do exposto, objetivou-se analisar os resultados da Triagem Auditiva Neonatal em recém-nascidos portadores de sífilis congênita.

Dessa forma, é possível estabelecer um plano para minimizar os danos causados através do encaminhamento rápido e adequados ao tratamento que a criança necessita. Para isso, é necessário o conhecimento prévio da equipe de profissionais da maternidade para orientar adequadamente os responsáveis e dar o apoio que eles precisam para adesão ao tratamento, pois se sabe que se esse não for realizado a tempo, os danos podem se tornar irreparáveis.

A motivação para a realização do presente estudo veio a partir da revisão bibliográfica demonstrar o quão prevalente são os fatores de risco que influenciam em alteração da Triagem Auditiva Neonatal, entre elas a sífilis congênita.

Apesar do fácil diagnóstico e de ser uma infecção curável, a sífilis ainda possui alta prevalência, principalmente em países subdesenvolvidos. Por isso a importância da preconização do Ministério da Saúde na realização de testes sorológicos na primeira consulta e repetição no início do terceiro trimestre e, quando diagnosticada, iniciar imediatamente o tratamento com Penicilina Benzatina concomitante ao parceiro, visando reduzir os riscos de reinfecção e, consequentemente, as chances de infectar o concepto e causar 
diversos problemas. Além disso, é de suma importância realizar as Triagens Neonatais para diagnosticar precocemente alterações que podem ser revertidas ou minimizadas a fim de causar o menor impacto possível na vida dessas crianças.

\section{METODOLOGIA}

Trata-se de um estudo retrospectivo, transversal observacional a partir do levantamento de dados no período de um ano de uma maternidade filantrópica do estado de Sergipe com o intuito de analisar o perfil epidemiológico dos resultados da triagem auditiva neonatal. A população utilizada na atual pesquisa consiste em recém-nascidos portadores de sífilis congênita que foram submetidos à Triagem Auditiva Neonatal e encaminhados ao ambulatório de sífilis do Hospital e Maternidade Santa Isabel no período de janeiro a dezembro de 2019.

Os dados foram compilados no programa Microsoft Office Excel versão 2016. Foram obtidas variáveis qualitativas nominais e variáveis quantitativas, onde a análise dos dados foi realizada de duas formas, descritiva e inferencial. Para as variáveis qualitativas a análise descritiva procedeu com a categorização dos dados e obtenção das respectivas frequências e percentuais, e para as variáveis quantitativas foram calculadas medidas de tendência central (média, mediana), variabilidade (desvio padrão) e posição (mínimo e máximo).

O presente estudo não causa danos ao paciente, pois serão analisados apenas os prontuários, não havendo contato físico entre a população estudada. Os pesquisadores garantem sigilo, confiabilidade e fidedignidade acerca dos dados estudados. O Termo de Consentimento Livre e Esclarecido (TCLE) não será necessário, pois trata-se de uma pesquisa retrospectiva em que apenas os prontuários serão analisados. 0 termo que contém a dispensa do TCLE foi encaminhado ao CEP.

\section{RESULTADOS}

Foram avaliados no total 117 recém-nascidos. A maioria é do sexo masculino ( $n=63 ; 53,8 \%$ ), com idade gestacional considerada a termo (nascidos a partir de 37 semanas), realizaram a triagem auditiva neonatal ( $n=60 ; 69,8 \%)$. Dos recém-nascidos que realizaram o teste, $63,3 \%(n=38)$ tiveram o resultado normal e $36,7 \%(n=22)$ tiveram alterações auditivas. Dos que apresentaram alteração auditiva, $54,5 \%$ estavam aguardando a realização do reteste, $36,4 \%$ tiveram seu reteste alterado e estavam aguardando um novo teste e apenas $9,1 \%$ apresentou reteste normal. O perímetro cefálico médio foi $34,2( \pm 1,5)$, variando de 30,5 a 38,0. Já em relação ao peso, apresentaram peso mínimo de 2080g e máximo de 4565g, com média de 3179g $( \pm 483,3)$ (Tabela 1).

Tabela 1: Estatística descritiva das características do recém-nascido.

\begin{tabular}{llllllll}
\hline Variável & $\mathbf{N}$ & Média & Mediana & Desvio Padrão & Intervalo Interquartil & Mínimo & Máximo \\
\hline Perímetro cefálico & 114 & 34,2 & 34,0 & 1,5 & 2,0 & 30,5 & 38,0 \\
Peso de nascimento & 117 & 3170,3 & 3115,0 & 483,3 & 590,0 & 2080,0 & 4565,0 \\
Apgar 1 $\mathbf{1}^{\circ}$ min & 109 & 8,7 & 9,0 & 1,0 & 0,0 & 3,0 & 10,0 \\
Apgar 5 $\mathbf{~}^{\circ}$ & 109 & 9,6 & 10,0 & 0,7 & 1,0 & 6,0 & 10,0 \\
\hline Variável/categorias & \multicolumn{5}{c}{ Frequência } \\
\hline
\end{tabular}




\begin{tabular}{lll}
\hline $\begin{array}{l}\text { Sexo do RN } \\
\text { Feminino }\end{array}$ & 54 & 46,2 \\
$\begin{array}{l}\text { Masculino } \\
\text { Idade Gestacional }\end{array}$ & 63 & 53,8 \\
$\begin{array}{l}\text { A termo } \\
\text { Prematuro }\end{array}$ & 90 & 93,8 \\
$\begin{array}{l}\text { Triagem auditiva neonatal } \\
\text { Não realizada }\end{array}$ & 6 & 6,3 \\
$\begin{array}{l}\text { Realizada } \\
\text { Resultado }\end{array}$ & 26 & 30,2 \\
$\begin{array}{l}\text { Alterado } \\
\text { Normal }\end{array}$ & 60 & 69,8 \\
Exame Alterado & & \\
$\begin{array}{l}\text { Aguardando reteste } \\
\text { Reteste normal }\end{array}$ & 22 & 36,7 \\
Reteste alterado & 38 & 63,3 \\
\hline
\end{tabular}

Verificou-se que foram realizadas em média $7( \pm 3,0)$ consultas pré-natal, com no mínimo de 0 e no máximo 15 consultas. Quando categorizada a variável número de consultas, 64,2\% ( $n=68$ ) realizaram 6 ou mais consultas. Em relação ao início do pré-natal, a maioria iniciou no $1^{\circ}$ trimestre e $73,5 \%(n=86)$ tinham a sífilis tratada (Tabela 2).

Tabela 2: Estatística descritivas das características obstétricas.

\begin{tabular}{|c|c|c|c|c|c|c|c|}
\hline Variável & $\mathbf{N}$ & Média & Mediana & Desvio Padrão & Intervalo Interquartil & Mínimo & Máximo \\
\hline $\mathrm{N}^{\circ}$ consultas pré-natal & 106 & 7 & 6 & 3 & 3 & 0 & 15 \\
\hline Variável/categorias & & Frequência & & & Porcentagem & & \\
\hline \multicolumn{8}{|l|}{ № Consultas } \\
\hline Menor que 6 & & 38 & & & 35.8 & & \\
\hline 6 ou mais & & 68 & & & 64.2 & & \\
\hline \multicolumn{8}{|l|}{ Início pré-natal } \\
\hline $1^{\circ}$ trimestre & & 53 & & & 60.8 & & \\
\hline $2^{\circ}$ trimestre & & 31 & & & 35.6 & & \\
\hline $3^{\circ}$ trimestre & & 3 & & & 3.4 & & \\
\hline \multicolumn{8}{|l|}{ Infecção no pré-natal } \\
\hline Sífilis não tratada & & 31 & & & 26.5 & & \\
\hline Sífilis tratada & & 86 & & & 73.5 & & \\
\hline
\end{tabular}

A maioria dos pacientes que apresentaram alterações auditivas eram do sexo feminino ( $n=12 ; 54,5 \%)$, com idade gestacional considerada normal (nascidos com 37 semanas ou mais) ( $n=15 ; 83,3 \%$ ). Em relação ao número de consultas realizadas no pré-natal que tiveram alterações auditivas, a maioria das mães fizeram menos de 6 consultas ( $n=13 ; 61,9 \%$ ) (Tabela 3 ).

Tabela 3: Associação entre os resultados do exame e as características do recém-nascido e obstétricas.

\begin{tabular}{|c|c|c|c|}
\hline \multirow{3}{*}{ Variável/Categorias } & \multicolumn{2}{|c|}{ Exame } & \multirow{3}{*}{ p-valor } \\
\hline & Alterado & Normal & \\
\hline & $\mathrm{N}(\%)$ & $\mathbf{N}(\%)$ & \\
\hline \multicolumn{4}{|l|}{ Sexo do RN } \\
\hline Feminino & $12(54,5)$ & $18(47,4)$ & 0,788 \\
\hline Masculino & $10(45,5)$ & $20(52,6)$ & \\
\hline \multicolumn{4}{|l|}{ Consultas } \\
\hline Menos que 6 consultas & $13(61,9)$ & $24(72,7)$ & 0,593 \\
\hline 6 ou mais consultas & $8(38,1)$ & $9(27,3)$ & \\
\hline \multicolumn{4}{|l|}{ Infecção } \\
\hline Sífilis / não tratada & $5(22,7)$ & $8(21,1)$ & 0,879 \\
\hline Sífilis / tratada & $17(77,3)$ & $30(78,9)$ & \\
\hline \multicolumn{4}{|l|}{ Idade Gestacional } \\
\hline Pré-termo & $3(16,7)$ & $3(10,3)$ & 0,662 \\
\hline A termo & $15(83,3)$ & $26(89,7)$ & \\
\hline
\end{tabular}


Nota-se que nenhumas das variáveis apresentaram diferença estatisticamente significativa ao nível de significância de 5\%. Usando a variável idade como exemplo, note que idade mediana das mães em que seus filhos apresentaram alterações auditivas é de 23 anos contra 24 anos das que tiveram o resultado normal, ou seja, não temos evidências suficientes em afirmar que idade das mães de RN com alteração auditiva é menor que aqueles sem alteração

Tabela 4: Associação entre os resultados do exame e as características do recém-nascido e obstétricas.

\begin{tabular}{|c|c|c|c|c|c|c|}
\hline Variável & $\mathbf{N}$ & Mediana & Intervalo Interquartil & Mínimo & Máximo & p-valor \\
\hline \multicolumn{7}{|c|}{ Idade Gestacional } \\
\hline Alterado & 22.0 & 39.0 & 1.0 & 35.0 & 41.0 & \multirow[t]{2}{*}{0.117} \\
\hline Normal & 38.0 & 39.0 & 0.0 & 35.0 & 40.0 & \\
\hline \multicolumn{7}{|l|}{ Peso RN } \\
\hline Alterado & 22.0 & 3012.5 & 420.0 & 2240.0 & 3890.0 & \multirow[t]{2}{*}{0.217} \\
\hline Normal & 38.0 & 3142.5 & 580.0 & 2175.0 & 4250.0 & \\
\hline \multicolumn{7}{|c|}{ Apgar $1^{\circ} \mathrm{min}$} \\
\hline Alterado & 22.0 & 9.0 & 1.0 & 6.0 & 9.0 & \multirow[t]{2}{*}{0.300} \\
\hline Normal & 38.0 & 9.0 & 0.0 & 5.0 & 9.0 & \\
\hline \multicolumn{7}{|c|}{ Apgar $5^{\circ} \mathrm{min}$} \\
\hline Alterado & 22.0 & 10.0 & 1.0 & 8.0 & 10.0 & \multirow[t]{2}{*}{0.663} \\
\hline Normal & 38.0 & 10.0 & 1.0 & 8.0 & 10.0 & \\
\hline \multicolumn{7}{|c|}{ Perímetro cefálico } \\
\hline Alterado & 22.0 & 33.3 & 2.0 & 31.0 & 37.0 & \multirow[t]{2}{*}{0.376} \\
\hline Normal & 38.0 & 34.0 & 1.5 & 31.0 & 37.0 & \\
\hline \multicolumn{7}{|c|}{$\mathbf{N}^{\circ}$ consultas pré-natal } \\
\hline Alterado & 22.0 & 6.0 & 4.0 & 3.0 & 12.0 & \multirow[t]{2}{*}{0.774} \\
\hline Normal & 38.0 & 6.0 & 3.0 & 1.0 & 12.0 & \\
\hline
\end{tabular}

\section{DISCUSSÃO}

Participaram do estudo todos (117) recém-nascidos que foram acompanhados no ambulatório de sífilis congênita do hospital ensino em Aracaju no ano de 2019. No presente estudo todos os neonatos que realizaram o teste tinham o diagnóstico de sífilis congênita. Cerca de 26,5\% das mães afirmaram não terem feito tratamento para essa patologia durante a gestação.

O índice de cobertura do PTAN foi de 69,2\%. Portanto, o caráter de universalidade do PTAN não foi alcançado, ou seja, a cobertura de $95 \%$ dos RN do hospital, como preconizado pelo Joint Committee On Infant Hearing $(\mathrm{JClH})$. A realização da TAN o quanto antes permite a otimização da intervenção, contribuindo para o desenvolvimento da criança. A identificação precoce das alterações auditivas possibilita a intervenção ainda no período crítico e ideal de estimulação da linguagem e da audição (NORTHERN et al., 1991).

A presente pesquisa apontou que $63,3 \%$ passaram na TAN, enquanto $36,7 \%$ falharam. É importante entender que o "passar" no teste equivale dizer que, no momento do teste, os resultados foram consistentes com respostas presentes nas EOAT, ou seja, compatível com integridade das células ciliadas externas. Ao longo do desenvolvimento infantil, perdas adquiridas decorrentes de: otite secretora, infecções, ototóxicos, causas genéticas ou traumáticas, podem gerar perdas auditivas permanentes (WIDEN et al., 2003).

O Comitê Brasileiro sobre Perdas Auditivas na Infância refere que a indicação ao reteste não deve ultrapassar 4\%. A presente pesquisa encontrou um índice de 36,7\%, inferindo um elevado índice de RN encaminhados para uma nova avaliação. Esse fato pode ser justificado pela possível presença de respiração ruidosa, ato de sucção ou ruído, fatores prejudiciais para captação das EOAT (RIBEIRO, 2001). 
Em relação ao pré-natal, $64,2 \%$ das mães realizam mais que seis consultas durante a gestação, atendendo aos requisitos do Ministério da Saúde. Idealmente, devem ser realizadas, no mínimo, seis consultas de pré-natal, sendo, preferencialmente, uma no primeiro trimestre, duas no segundo trimestre e três no terceiro trimestre da gestação (BRASIL, 2006). O acompanhamento pré-natal, estratégia importante de cuidados preventivos em gestantes e crianças, é capaz de orientar a promoção da saúde e do bem-estar, além de oportunizar o tratamento de problemas que podem afetar as mães e seus filhos nesse período (ALMEIDA et al., 2007). No Brasil, o número de consultas pré-natais por mulheres que realizam partos pelo Sistema Único de Saúde (SUS) vem aumentando. Por exemplo, em 1995, ocorriam 1,2 consultas por parto, e em 2005, ocorreram 5,45 consultas por parto. No entanto, esse indicador apresenta diferenças regionais significantes. Em 2003, o percentual de nascidos de mães que fizeram sete ou mais consultas foi menor no Norte e Nordeste (BRASIL, 2006).

O presente estudo teve uma taxa de $6,3 \%$ de partos prematuros. Desses, $50 \%$ tiveram alteração no exame de triagem auditiva neonatal. Em relação ao nascimento pré-termo, encontram-se na literatura estudos com resultados diversos: alguns deles com percentagens próximas do observado no hospital ensino em que foi realizado o estudo, com taxa de 4,0\% e 4,75\% (BORGES et al., 2006; WROBLEWSKA et al., 2005). Em contrapartida, foi encontrado também, em outro estudo, percentagem elevada $(39,1 \%)$ de crianças apresentando este indicador de risco (TIENSOLI et al., 2007). Esse último estudo, diferente da atual pesquisa, foi realizado em maternidade de referência para gestações e partos de alto risco e, provavelmente por isso, encontrou percentagens elevadas.

Neste estudo em torno de $54,4 \%$ dos testes alterados foram encontrados em RN do sexo feminino. Além disso, a média de peso ao nascimento dos RN que tiveram a TAN alterada foi de $3012.5 \mathrm{~g}$, tendo a mínima de $2240 \mathrm{~g}$ e a máxima de $3890 \mathrm{~g}$. Fatores como baixo peso ao nascer e índice de Apgar baixo podem causar aumento da morbidade neonatal e são responsáveis, em muitos casos, por atraso no desenvolvimento global risco (TIENSOLI et al., 2007). O índice de Apgar tem a finalidade de verificar de forma rápida o estado clínico do neonato e identificar aqueles que necessitam de assistência, avaliar riscos e prevenir sequelas. Ele consta de cinco parâmetros: frequência cardíaca, respiração, tônus muscular, irritabilidade reflexa e coloração da pele do recém-nascido. Numa escala de 0 a 10 , se o seu valor for menor que 7 , será diagnosticada hipóxia fetal (CORRÊA et al., 2006). O citado estudo encontrou nos RN que tiveram alterações na TAN, o menor apgar no $1^{\circ}$ minuto de 6 e o maior de 10 . Enquanto no $5^{\circ}$ minuto, o menor Apgar encontrado foi de 8 e o maior de 10. Isso reflete o fato da maternidade em questão ser referência para partos de baixo risco.

\section{CONCLUSÕES}

Por trata-se de uma população de risco para desenvolvimento de alterações auditivas, o presente estudo demonstrou um alto valor de alterações dos testes realizados para Triagem Auditiva Neonatal. Outro fator importante para alteração na TAN foi a prematuridade, pois em $50 \%$ dos casos de RN prematuros houve a alteração no exame. Portanto, a melhor forma de prevenir qualquer tipo de alteração ainda é fazer um pré- 
natal adequado, tratando todas as intercorrências que ocorra, inclusive infecções congênitas.

\section{REFERÊNCIAS}

ALMEIDA, M. F.; ALENCAR, G. P.; NOVAES, H. M. D.; FRANÇA JÚNIOR, I.; SIQUEIRA, A. A. F.; CAMPBELL, O. M. R.. Fatores de risco para mortes fetais anteparto no Município de São Paulo. Brasil. Revista de Saúde Pública, v.41, p.35-43. 2007. DOI: https://doi.org/10.1590/\$0034-89102007000100006

AZEVEDO, M. F.; FERREIRA, L. P.; BEFI-LOPES, D. M.; LIMONGI, S. C. O.. Tratado de fonoaudiologia. Triagem Auditiva Neonatal. São Paulo: Rocca, 2004.

BORGES, C. A. B.; MOREIRA, L. M. O.; PENA, G. M.; FERNANDES, F. R.; BORGES, B. C. B.; OTANI, B. H.. Triagem Auditiva Neonatal Universal. Arq. Int. Otorrinolaringologia. São Paulo, v.10, n.1, p.28-34, 2006.

BRASIL. Pré-natal e puerpério: atenção qualificada e humanizada. Brasília, 2006.

\section{BRASIL. Diretrizes de atenção da Triagem Auditiva} Neonatal. Brasília, 2012.

CORRÊA, R. R. M.; SALGE, A. K. M.; RIBEIRO, G. A.; FERRAZ, M. L. F.; REIS, M. A.; CASTRO, E. C. C.. Alterações anatomopatológicas da placenta e variações do índice de Apgar. Revista Brasileira de Saúde. Mater. Infant., Recife, v.6, n.2, p.239-43, 2006. DOI:

http://dx.doi.org/10.1590/S1519-38292006000200012

DAHLE, A. J.; FOWLER, K. B.; WRIGHT, J. D.; BOPPANA, S. B.; BRITT, W. J.; PASS, R. F.. Longitudinal investigation of hearing disorders in children with congenital cytomegalovirus. Journey of the American Academic of Audiology, v.11, n.5, p.283-90, 2000.

NORTHERN, J. L.; DOWNS, M. P.. Behavioral hearing testing of children. Hearing in children. Baltimore, 1991.

PÁDUA, F. G. M.. Triagem Auditiva Neonatal: um Desafio para sua Implantação. Revista Brasileira de Otorrinolaringologia, São Paulo, v.9, n.3 p.189-94, 2005.
RIBEIRO, F. M.. Programa de Triagem Auditiva Neonatal. In: HERNANDEZ, A. M.; MARCHESAN, I.. Atuação

fonoaudiológica no ambiente hospitalar. Rio de Janeiro: Revinter, 2001. p.152-5.

RUGGIERI, M.; LICHTIG, I.; MARONE, S. A. M.. Recémnascidos gerados por mães com alto risco gestacional: estudo das emissões otoacústicas produtos de distorção e do comportamento auditivo. Revista Brasileira de Otorrinolaringologia, v.68, n.2 p.230-7,2002.

SANTOS, P. H.; IAZZETTI, A. V.. Citomegalia congênita e perinatal. Pediatr Mod., v.36, n.12, p.805-27, 2000.

TIENSOLI, L. O.; GOULART, L. M. H. F.; RESENDE, L. M.; COLOSIMO, E. A.. Triagem auditiva em hospital público de Belo Horizonte, Minas Gerais, Brasil: Deficiência auditiva e seus fatores de risco em neonatos e lactentes. Caderno de Saúde Pública, v.23, n.6, p.1431-41, 2007. DOI: https://doi.org/10.1590/S0102-311X2007000600018

VIEIRA, E. P.; TOCHETTO, T. M.; PEDROSO, F. S.. Indicadores de risco para a deficiência auditiva infantil: infecções congênitas. Fono Atual, n.8, v.32, p.61-7, 2005.

WEBER, B. A.; DIEFENDORF, A.. Triagem auditiva neonatal. In: MUSIEK, F. R.; RINTELMANN, W. F.. Perspectivas atuais em avaliação auditiva. São Paulo: Manole, 2001. p.323-38.

WIDEN, J. E.; BULL, W.; FOLSOM, R. C.. Newborn Hearing Screening: What it means for providers of early intervention services. LWW/IYC, v.16, n.3 p.249-57, 2003. DOI: http://doi.org/10.1097/00001163-200307000-00007

WROBLEWSKA-SENIUK, K.; CHOJNACKA, K.; PUCHER, B.; SZCZAPA, J.; GADZINOWSKI, J.; GRZEGOROWSKI, M.. The results of newborn hearing screening by means of transient evoked otoacoustic emissions. Int. J. Pediatr.

Otorhinolaryngol, v.69, n.10, p.351-1357, 2005. DOI:

http://doi.org/10.1016/i.ijporl.2005.03.023

A CBPC - Companhia Brasileira de Produção Científica (CNPJ: 11.221.422/0001-03) detém os direitos materiais desta publicação. Os direitos referem-se à publicação do trabalho em qualquer parte do mundo, incluindo os direitos às renovações, expansões e disseminações da contribuição, bem como outros direitos subsidiários. Todos os trabalhos publicados eletronicamente poderão posteriormente ser publicados em coletâneas impressas sob coordenação da Sustenere Publishing, da Companhia Brasileira de Produção Científica e seus parceiros autorizados. Os (as) autores (as) preservam os direitos autorais, mas não têm permissão para a publicação da contribuição em outro meio, impresso ou digital, em português ou em tradução. 